\section{Benefit of intermediate-dose cytarabine-containing induction in molecular subgroups of acute myeloid leukemia}

The outcome of acute myeloid leukemia (AML) is affected by disease characteristics as well as treatment regimens. ${ }^{1-3}$ In the CALGB8525 trial, patients with core binding factor (CBF)-positive leukemia benefited from consolidation with a high dose of cytarabine. ${ }^{4}$ More recently, high-dose daunorubicin $\left(60-90 \mathrm{mg} / \mathrm{m}^{2}\right)$ has become widely used. ${ }^{5,6}$ High-dose daunorubicin confers a favorable prognosis for patients with NPM1 mutations. $1,7,8$

Higher-dose cytarabine was also introduced into AML induction therapy. ${ }^{3,9}$ Recently, we investigated the role of intermediate-dose cytarabine in induction therapy of AML and found that the introduction of intermediatedose cytarabine, combined with daunorubicin and omacetaxine mepesuccinate, improved outcomes in patients with new-diagnosed AML. ${ }^{2}$ Overall, 591 patients aged 15 to $<55$ years with de novo newly-diagnosed AML were enrolled in our study, registered at www.chictr.org.cn (trial identifier: ChiCTR-TRC-10001202), as described in detail in our previous report. ${ }^{2}$ The characteristics of the patients at study entry were included in that report. ${ }^{2}$ The distribution of the cytogenetic and mutation subgroups is shown in Online Supplementary Table S1. Eligible patients were randomly-assigned to conventional-dose cytarabine (100 $\mathrm{mg} / \mathrm{m}^{2} /$ day on days $1-7$ as a 12 -h intravenous infusion) or intermediate-dose cytarabine $\left(100 \mathrm{mg} / \mathrm{m}^{2} /\right.$ day on days 1 4 as a 12 -h intravenous infusion and $1 \mathrm{~g} / \mathrm{m}^{2}$ every $12 \mathrm{~h}$ as a 3 -h intravenous infusion on days 5-7). Patients also received daunorubicin $\left(40 \mathrm{mg} / \mathrm{m}^{2} /\right.$ day on days $\left.1-3\right)$ and omacetaxine mepesuccinate $\left(2 \mathrm{mg} / \mathrm{m}^{2} /\right.$ day on days $\left.1-7\right)$ (see the Online Supplementary Materials and Methods for details). Here we updated results with longer follow-up and focused on the benefit of intermediate-dose cytarabine induction in molecular subgroups of AML. The median follow-up time of survivors in the current report was 70 months (range, 5-115 months).

In total, 107 of 591 patients underwent allogeneic transplantation in first complete remission (CR1). With longer follow-up, the induction regimen with intermediate-dose cytarabine improved relapse-free survival (RFS), event-free survival (EFS), and overall survival (OS) in the entire cohort compared with outcomes achieved with conventional-dose cytarabine (Online Supplementary Figure S1), as before. ${ }^{2}$ The intermediate-dose cytarabine still improved RFS, EFS, and OS in patients with intermediate-risk cytogenetics (Online Supplementary Table S2). Intermediate-dose cytarabine produced better RFS and EFS in patients with favorable cytogenetics in univariate and multivariable analyses, as shown in Online Supplementary Table S2 and Online Supplementary Figure $S 2$. However, intermediate-dose cytarabine was not associated with better OS, despite the longer follow-up, in patients with favorable cytogenetics. We were unable to determine the benefit of intermediate-dose cytarabine in the adverse cytogenetic cohorts due to small sample sizes.

Overall, there were 75 patients with CEBPA double mutations $(C E B P A \mathrm{dm})$ in our cohort, including 32 in the conventional-dose group and 43 in the intermediate-dose group. Intermediate-dose cytarabine did not increase the complete remission rate in patients with CEBPAdm $(95 \%$ and $100 \%$ in the intermediate-dose and conventionaldose cytarabine groups, respectively; $P=0.504$ ). Intermediate-dose cytarabine did, however, produce bet- ter RFS and EFS rates and showed a marked tendency to improve the OS of patients with CEBPAdm in both univariate and multivariable analyses, as shown in Online Supplementary Table S2. Five-year RFS, EFS, and OS rates were $85 \%, 81 \%$, and $88 \%$ in the intermediate-dose compared with $56 \%, 56 \%$, and $68 \%$ in the conventionaldose group, respectively (Figure 1). In total, 13 of 75 $(17 \%)$ patients with CEBPAdm AML underwent allogeneic transplantation in CR1, including five of $32(16 \%)$ in the conventional-dose group and eight of $43(19 \%)$ in the intermediate-dose group. To analyze results in the absence of any possible contributory effect of transplantation, patients were censored at the time of transplantation in CR1. Patients with CEBPAdm AML exposed to intermediate-dose cytarabine achieved an increase in 5year RFS, censored at the date of transplantation, from $56 \%$ to $83 \%$ (hazard ratio [HR], $0.313 ; 95 \%$ confidence interval [95\% CI]: $0.119-0.824$; Wald $P=0.019$ ) (Online Supplementary Figure S3). Intermediate-dose cytarabine showed a tendency to increase EFS and OS rates, censored at the date of transplantation, from $58 \%$ to $79 \%$ (HR, 0.420; 95\% CI: 0.174-1.013; Wald $P=0.053$ ), and from $74 \%$ to $89 \%$ (HR, 0.398; 95\% CI: $0.133-1.187$; Wald $P=0.099$ ), respectively (Online Supplementary Figure $S 3)$. We found a significant interaction between treatment assignment and CEBPAdm status in RFS $(P=0.042)$, but not EFS $(\mathrm{P}=0.184)$ or OS $(\mathrm{P}=0.119)$. The hazard ratios for relapse or death of CEBPAdm AML compared with other types of AML were 0.298 (95\% CI: 0.130-0.682; Wald $P=0.004)$ in the intermediate-dose cytarabine group and 0.829 (95\% CI: $0.473-1.453$; Wald $P=0.513$ ) in the conventional-dose cytarabine group (Figure 1). The data indicated that the favorable RFS of patients with CEBPAdm AML depended on treatment assignment. After adjusting for the presence of FLT3-ITD and transplantation in CR1, the interaction between treatment assignment and CEBPAdm status still existed for RFS $(P=0.042)$, but not for EFS $(P=0.215)$ or OS $(P=0.148)$.

The OS and RFS rates of AML patients with CEBPAdm are approximately $54 \%-63 \%$ and $44-48 \%$, respectively. ${ }^{10-}$ ${ }^{13}$ However, relapsed patients with CEBPAdm have a favorable outcome after reinduction followed by allogeneic transplantation. Schlenk et al. proposed both strategies, allogeneic or autologous transplantation in CR1 versus intensive chemotherapy in CR1, and reinduction followed by allogeneic transplantation in the case of relapse. ${ }^{13}$ We demonstrated that CEBPAdm AML patients receiving intermediate-dose cytarabine had a remarkable increase in RFS as well as in RFS rates censored at the date of allogeneic transplantation. This indicated that more patients would not relapse and did not need transplantation after intermediate-dose cytarabine induction therapy.

Overall, there were 131 patients with RUNX1RUNX1T1 in our cohort, including 60 in the conventional-dose group and 71 in the intermediate-dose group. Intermediate-dose cytarabine did not increase the complete remission rate in patients with RUNX1-RUNX1T1 compared to that in patients treated with conventionaldose cytarabine $(97 \%$ and $93 \% ; P=0.528)$. However, intermediate-dose cytarabine produced better RFS and EFS and showed a marked tendency to improve OS in patients with RUNX1-RUNX1T1 in both univariate and multivariable analyses, as shown in Online Supplementary Table S2. The 5-year RFS, EFS, and OS rates in patients with RUNX1-RUNX1T1 AML were $72 \%, 70 \%$, and $74 \%$ in the intermediate-dose cytarabine group compared to $56 \%, 52 \%$, and $58 \%$ in the conventional-dose group, respectively (Figure 2). There was no interaction between 
A

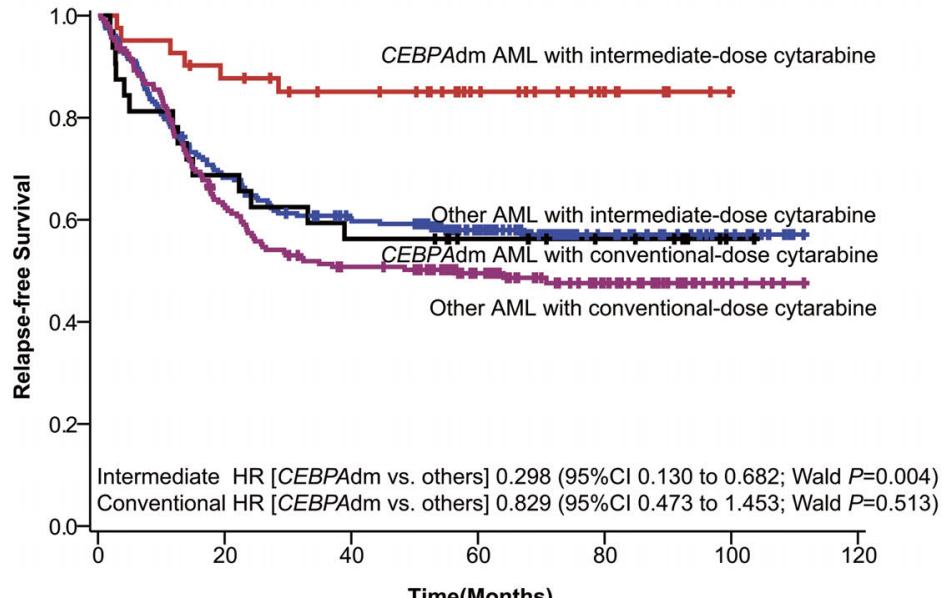

Number at risk CEBPAdm AML Intermediate 41 CEBPAdm AML conventional 32 Other AML intermediate 202 Other AML conventional $\quad 187$

$\begin{array}{ll}35 & 30 \\ 22 & 18 \\ 137 & 114 \\ 115 & 89\end{array}$

\section{Time(Months)}

$\begin{array}{cccc}21 & 10 & 0 & 0 \\ 13 & 8 & 1 & 0 \\ 81 & 33 & 11 & 0 \\ 64 & 33 & 7 & 0\end{array}$

\section{B}

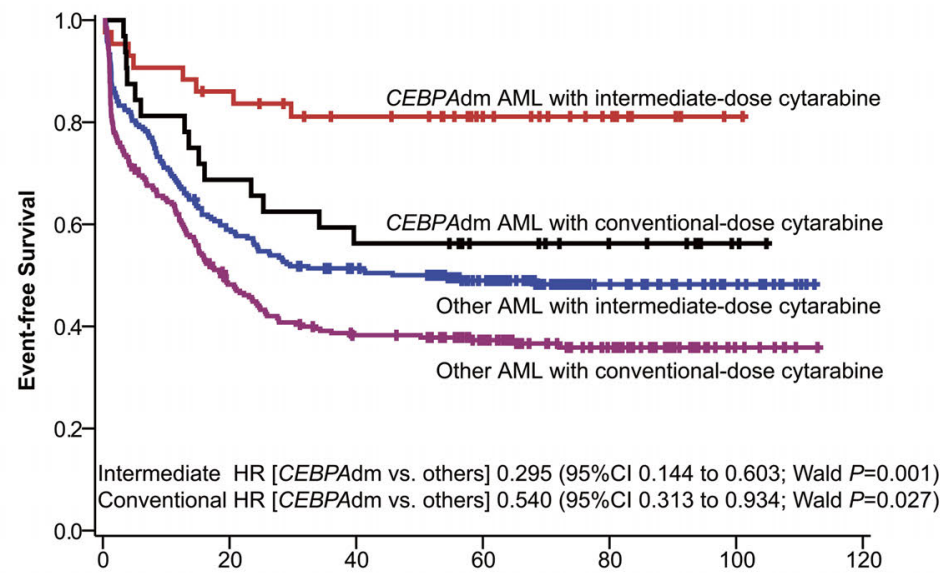

Number at risk CEBPAdm AML Intermediate 43 CEBPAdm AML conventional 32 Other AML intermediate $\quad 239$ Other AML conventional $\quad 250$

\begin{tabular}{lccccc}
\multicolumn{5}{c}{ Time(Months) } \\
36 & 30 & 21 & 12 & 1 & 0 \\
22 & 18 & 13 & 8 & 2 & 0 \\
140 & 84 & 84 & 34 & 12 & 0 \\
117 & 68 & 68 & 35 & 7 & 0
\end{tabular}

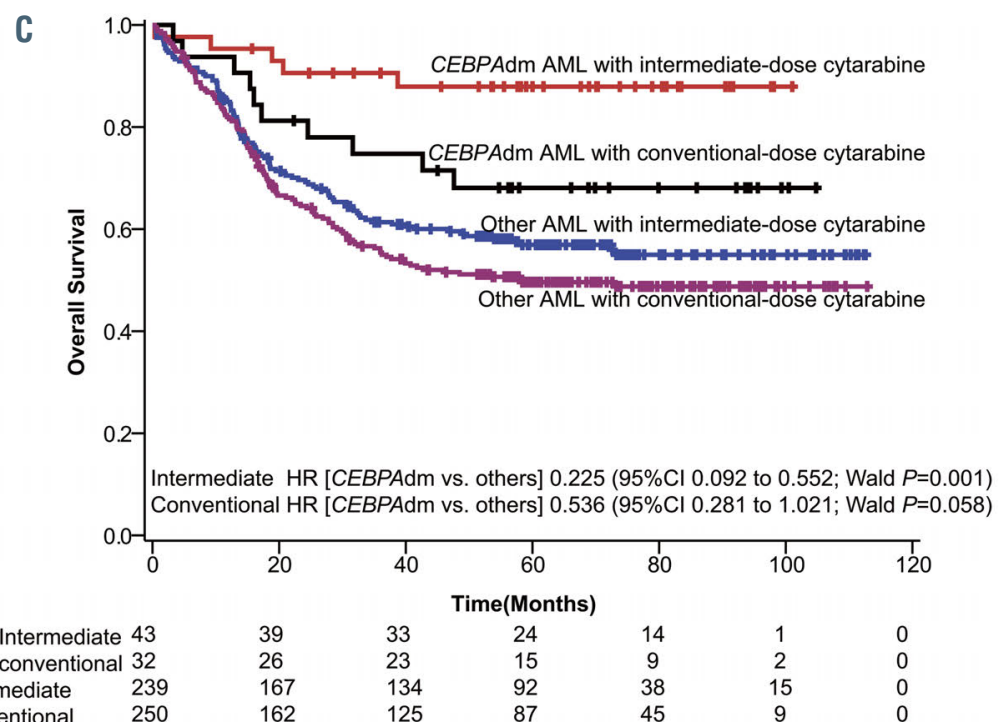

Figure 1. Patients with acute myeloid leukemia with CEBPA double mutations had more favorable outcomes only when treated with intermediate-dose cytarabine induction. (A) Relapse-free survival, (B) eventfree survival, and $(C)$ overall survival are shown for patients with CEBPA double mutations and other types of acute myeloid leukemia by receipt of intermediate-dose or conventional-dose cytarabine induction. AML: acute myeloid leukemia: HR: hazard ratio; $95 \% \mathrm{Cl}$ : $95 \%$ confidence interval.

Number at risk

CEBPAdm AML Intermediate CEBPAdm AML conventional 32

Other AML intermediate 250
167
162 
A

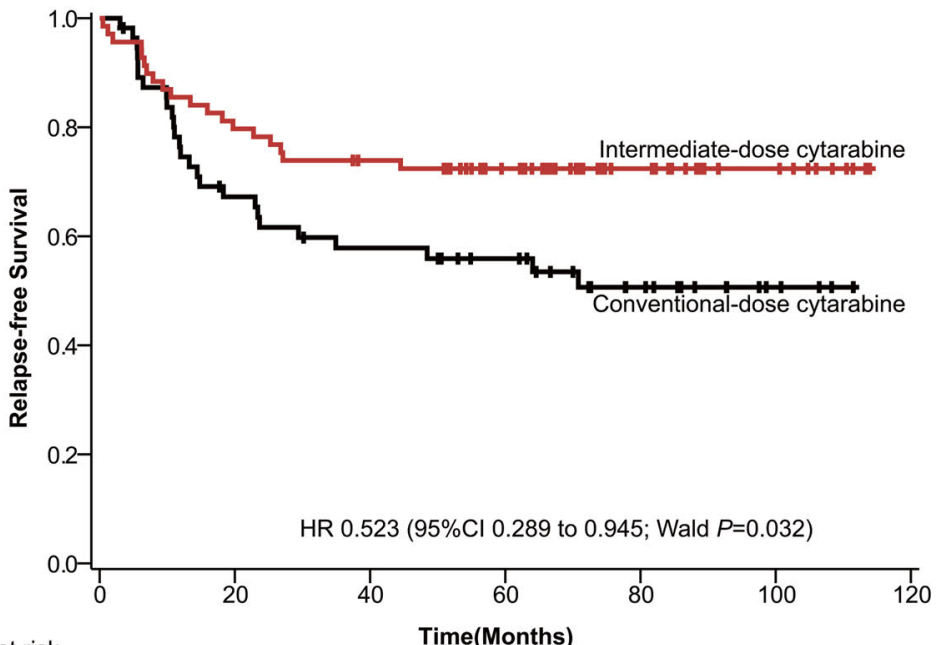

Number at risk

Intermediate

Conventiona

$69 \quad 55 \quad 49$

Time(Months)
38

25

$\begin{array}{lll}20 & 9 & 0 \\ 13 & 4 & 0\end{array}$

\section{B}

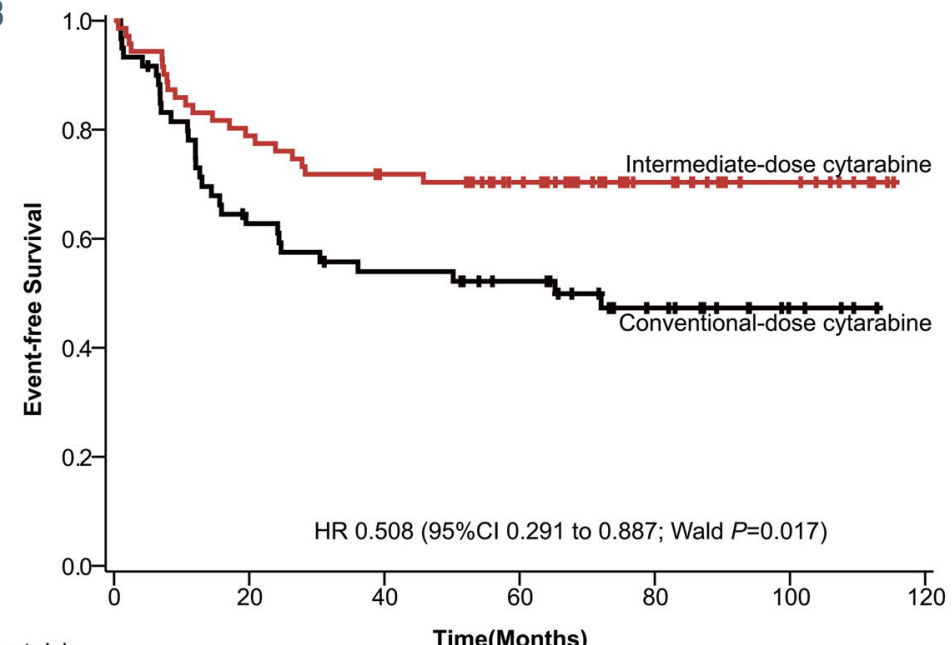

Number at risk

Intermediate

$\begin{array}{lllllll}71 & 56 & 49 & 39 & 20 & 9 & 0 \\ 60 & 36 & 30 & 25 & 13 & 4 & 0\end{array}$

Time(Months)

Conventiona

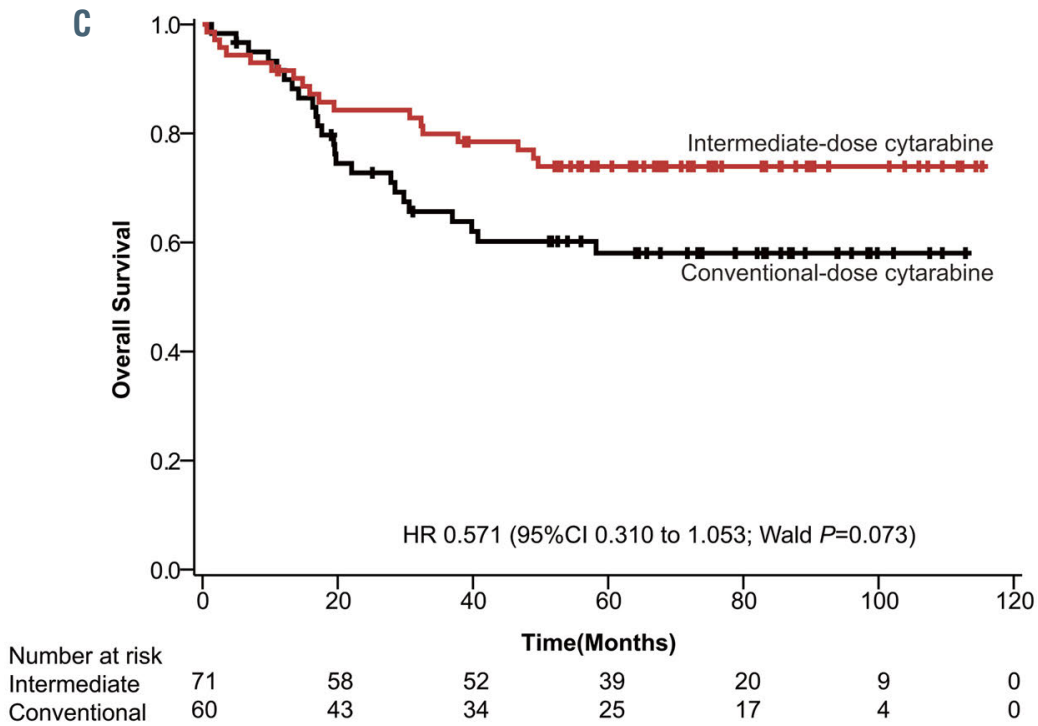

Figure 2. Outcomes of patients with RUNX1-RUNX1T1 acute myeloid leukemia by treatment assignment. (A) Relapse-free survival, (B) event-free survival, and (C) overall survival. HR: hazard ratio; 95\% $\mathrm{Cl}$ : 95\% confidence interval. 
the treatment assignment and RUNX1-RUNX1T1 status (RFS: $P=0.300$; EFS: $P=0.383$; OS: $P=0.391$ ). All patients with $C B F B-M Y H 11$ AML achieved complete remission after both intermediate-dose and conventional-dose cytarabine. We were unable to determine the impact of intermediate-dose cytarabine in patients with $C B F B$ MYH11 AML since there were only 33 patients with $C B F B-M Y H 11$ in our cohort.

In this subgroup analysis of our trial, our data suggested
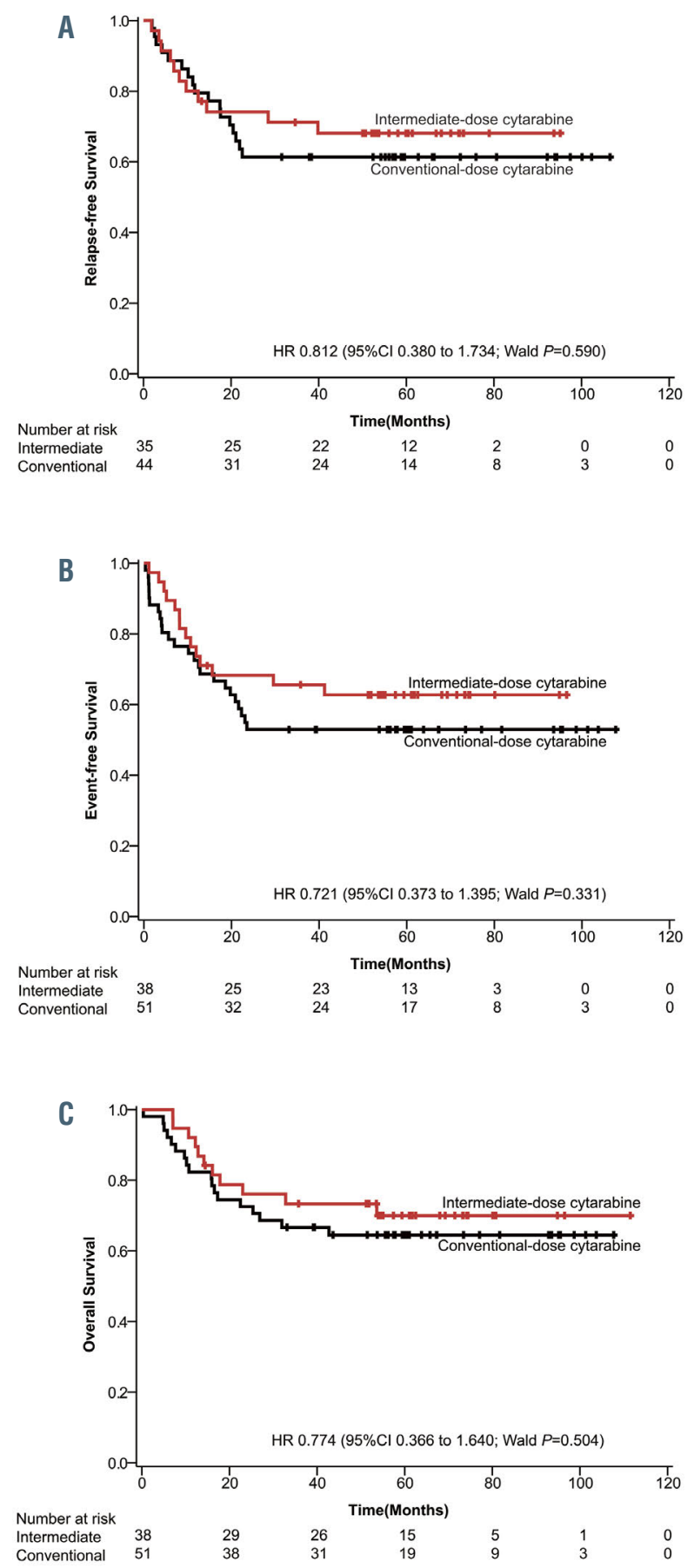

that AML patients with RUNX1-RUNX1T1 benefited from intermediate-dose cytarabine induction. Previous reports also indicated that a higher dose of cytarabine improved the outcome in patients with RUNX1-RUNX1T1 AML. ${ }^{14,15}$ Hence, all these data suggest that an induction regimen with an intensified dose of cytarabine benefits patients with RUNX1-RUNX1T1 AML.

There were a total of 89 patients with NPM1 mutations, regardless of FLT3-ITD mutation status, in our cohort,
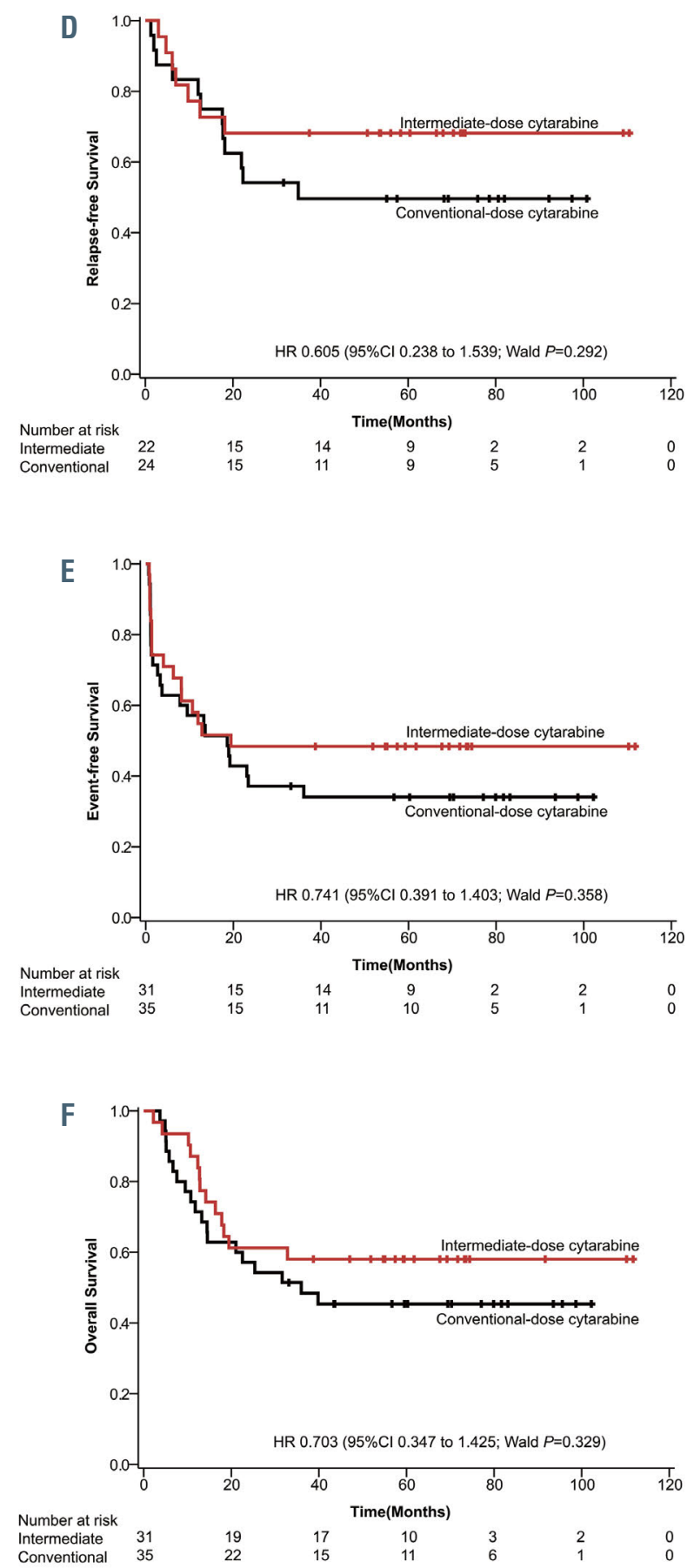
including 51 in the conventional-dose group and 38 in the intermediate-dose group. There were 66 patients with FLT3-ITD mutations, regardless of NPM1 mutations, including 35 in the conventional-dose group and 31 in the intermediate-dose group. Intermediate-dose cytarabine did not increase the complete remission rate or improve RFS, EFS, or OS compared to conventional-dose cytarabine in patients with NPM1 or FLT3-ITD mutations, as shown in Online Supplementary Table S2. In patients with NPM1 mutations, the 5-year RFS, EFS, and OS rates were $68 \%$, $63 \%$, and $70 \%$ in the intermediate-dose cytarabine group compared to $61 \%, 53 \%$, and $65 \%$ (Figure 3A-C), respectively, in the conventional-dose group. In patients with FLT3-ITD mutations, the 5-year RFS, EFS, and OS rates were $68 \%, 48 \%$, and $58 \%$ in the intermediate-dose cytarabine group compared to $50 \%, 34 \%$, and $45 \%$ (Figure 3DF), respectively, in the conventional-dose group. We then investigated the impact of intermediate-dose cytarabine in NPM1 $1^{+} / F L T 3-\mathrm{ITD}^{-}, \mathrm{NPM1}^{+} / \mathrm{FLT3}^{-} \mathrm{ITD}^{+}$, and NPM1-/FLT3$\mathrm{ITD}^{+}$subgroups. Intermediate-dose cytarabine did not increase complete remission rate or improve RFS, EFS, or OS compared to conventional-dose cytarabine in all these subgroups, as shown in Online Supplementary Table S3.

Death rates within 30 days were similar in the intermediate- and conventional-dose cytarabine induction cohorts. ${ }^{2}$ There were no significant differences in RFS, OS, cumulative incidence of relapse or cumulative incidence of death in complete remission between the consolidation regimens even with longer follow-up (data not shown). With inclusion of the second randomization in multivariable analyses, the conclusions regarding outcomes depending on induction treatment were not modified by the second randomization, as shown in Online Supplementary Table S4, except that the OS in the intermediate cytogenetic-risk group was not significantly different, but with a trend, and no difference in EFS in the poor cytogenetic-risk group.

In this subgroup analysis with updated follow-up, we demonstrated that AML patients with CEBPAdm and RUNX1-RUNX1T1 might benefit from intermediate-dose cytarabine induction. AML patients with CEBPAdm had a more favorable RFS than others only when treated with intermediate-dose cytarabine induction. Intermediate-dose cytarabine did not, however, improve outcomes in AML patients with NPM1 or FLT3-ITD mutations. Luskin et al. suggested that anthracycline dose intensification induction conferred a favorable prognosis for AML patients with NPM1 mutations. ${ }^{7}$ These data indicate that AML patients with different mutations might benefit from intensified doses of different drugs. Recently, novel drugs, such as gemtuzumab ozogamicin, FLT3 inhibitors and so on, are being used in clinical practice. Prospective trials would be needed to confirm the benefit of induction with intermediate-dose cytarabine, especially when novel drugs are used.

Hui Wei, ${ }^{1,2,3}$ Chunlin Zhou, ${ }^{3}$ Dong Lin, ${ }^{3}$ Bingcheng Liu, ${ }^{3}$ Yan Li, Xingli Zhao, ${ }^{3}$ Shuning Wei, ${ }^{3}$ Benfa Gong, ${ }^{3}$ Kaiqi Liu, ${ }^{3}$ Xiaoyuan Gong, ${ }^{3}$ Yuntao Liu, ${ }^{3}$ Guangji Zhang, ${ }^{3}$ Jiayuan Chen, Junping Zhang, Jinging Jin, ${ }^{3}$ Shaowei Qiu, ${ }^{3}$ Runxia $\mathrm{Gu}^{3}{ }^{3}$ Ying Wang, ${ }^{2,3}$ Yingchang $\mathrm{Mi}^{1,2,3}$ and Jianxiang Wang ${ }^{1,2,3}$

${ }^{1}$ State Key laboratory of Experimental Hematology; ${ }^{2}$ National Clinical Research Center for Blood Diseases and ${ }^{3}$ Leukemia Center, Institute of Hematology and Blood Diseases Hospital, Chinese Academy of Medical Sciences, Tianjin, China

Correspondence:

JIANXIANGWANG -wangix@ihcams.ac.cn

doi:10.3324/haematol.2020.267526

Received: July 19, 2020.
Accepted: September 15, 2020.

Pre-published: September 28, 2020.

Disclosures: no conflicts of interest to disclose.

Contributions: JW and HW contributed to the study design; $J W, H W$, and JC were involved in analyzing and interpreting the data; $J W$ and $H W$ wrote the report. All authors were involved in the collection and assembly of clinical data. $H W, D L, C Z, B L, S Q, R G, Y L$, $X Z, S W, B G, K L, X G, Y L i u, G Z, J Z, J J$, YW and YM provided the study materials or patients. All authors reviewed the report and approved the final version.

Funding: this study was supported in part by funds from: the State Key Program of National Natural Science of China (81830005) and the CAMS Innovation Fund for Medical Sciences (2019-I2M-2-009) to JW; the National Key Research and Development Program of China (2019YFC0840605) to YM; and the National Natural Science Foundation of China (81670159) and Tianjin Natural Science Foundation (18JCZDJC45000) to HW. The funders had no role in the study design, analyses, or decision to publish.

\section{References}

1. Fernandez HF, Sun Z, Yao X, et al. Anthracycline dose intensification in acute myeloid leukemia. N Engl J Med. 2009;361(13):1249-1259.

2. Wei H, Wang Y, Gale RP, et al. Randomized trial of intermediate-dose cytarabine in induction and consolidation therapy in adults with acute myeloid leukemia. Clin Cancer Res. 2020;26(13):3154-3161

3. Willemze R, Suciu S, Meloni G, et al. High-dose cytarabine in induction treatment improves the outcome of adult patients younger than age 46 years with acute myeloid leukemia: results of the EORTCGIMEMA AML-12 trial. J Clin Oncol. 2014;32(3):219-228.

4. Mayer RJ, Davis RB, Schiffer CA, et al. Intensive postremission chemotherapy in adults with acute myeloid leukemia. Cancer and Leukemia Group B. N Engl J Med. 1994;331(14):896-903.

5. Dohner H, Estey E, Grimwade D, et al. Diagnosis and management of AML in adults: 2017 ELN recommendations from an international expert panel. Blood. 2017;129(4):424-447.

6. NCCN clinical practice guidelines in oncology acute myeloid leukemia Version 3.2020. http://www.nccn.org. MS28-MS34.

7. Luskin MR, Lee JW, Fernandez HF, et al. Benefit of high-dose daunorubicin in AML induction extends across cytogenetic and molecular groups. Blood. 2016;127(12):1551-1558.

8. Lee JH, Joo YD, Kim H, et al. A randomized trial comparing standard versus high-dose daunorubicin induction in patients with acute myeloid leukemia. Blood. 2011;118(14):3832-3841.

9. Bishop JF, Matthews JP, Young GA, et al. A randomized study of highdose cytarabine in induction in acute myeloid leukemia. Blood. 1996; 87(5):1710-1717.

10. Green CL, Koo KK, Hills RK, et al. Prognostic significance of CEBPA mutations in a large cohort of younger adult patients with acute myeloid leukemia: impact of double CEBPA mutations and the interaction with FLT3 and NPM1 mutations. J Clin Oncol. 2010; 28(16):2739-2747

11. Taskesen E, Bullinger L, Corbacioglu A, et al. Prognostic impact, concurrent genetic mutations, and gene expression features of AML with CEBPA mutations in a cohort of 1182 cytogenetically normal AML patients: further evidence for CEBPA double mutant AML as a distinctive disease entity. Blood. 2011;117(8):2469-2475.

12. Wouters BJ, Lowenberg B, Erpelinck-Verschueren CA, et al. Double CEBPA mutations, but not single CEBPA mutations, define a subgroup of acute myeloid leukemia with a distinctive gene expression profile that is uniquely associated with a favorable outcome. Blood. 2009;113(13):3088-3091.

13. Schlenk RF, Taskesen E, van Norden Y, et al. The value of allogeneic and autologous hematopoietic stem cell transplantation in prognostically favorable acute myeloid leukemia with double mutant CEBPA. Blood. 2013;122(9):1576-1582.

14. Burnett AK, Hills RK, Russell N, et al. Reasons for survival improvement in core binding factor AML: a 25 year analysis of the UK MRC/NCRI AML trials. Blood. 2013;122(21):358.

15. Borthakur G, Kantarjian H, Wang X, et al. Treatment of core-bindingfactor in acute myelogenous leukemia with fludarabine, cytarabine, and granulocyte colony-stimulating factor results in improved eventfree survival. Cancer. 2008;113(11):3181-3185. 